received prehospital IV fluid (98.2\% vs. $81.9 \%)$. From univariate analysis, patients receiving prehospital IV fluid were less likely to survive (OR 0.08; 95\% CI: 0.05-0.14 p-value < 0.00001) after adjusted for other variables (age, sex, mechanism of injury, Glasgow Coma Scale, initial blood pressure and Injury Severity Score), prehospital IV fluid did not decrease survival (OR 0.85 ; 95\% CI: 0.35-2.07 p-value $=0.735$ ). However subgroup analysis in patients with hypotension at scene, prehospital IV fluid significantly decreased survival to discharge (OR 0.16; 95\% CI: $0.04-$ 0.76 p-value $=0.021$.

Conclusions Prehospital IV fluid did not increase survival in trauma victims and it may be harmful in hypotensive patients. The routine use of prehospital IV fluid administration for all trauma patients should be considered.

\section{COMPLEX THERAPY FOR TREATMENT OF OSTEOPOROSIS}

${ }^{1}$ Rustam Talishinskiy, ${ }^{2}$ Kamalya Rustamova. ${ }^{1}$ Scientific Research Institute of Traumatology and Orthopaedics, Azerbaijan; ${ }^{2}$ Scientific Research Institute of Obstetrics and Gynaecology, Azerbaijan

\subsection{6/injuryprev-2016-042156.788}

Background Osteoporosis is system disease of skeletal system with loosening of bone tissue mass and microarchitecture disorders, which follow to bone's fragility and high risk of fractures.

Statistical data make it possible to realise the level problem on Azerbaijan Republic. Population group of persons older than 50 y.o. increase, it was $19 \%$ of whole population in 2010, it consist 1700000 .

Specialist of Obstetrics and Gynaecology Institute of Azerbaijan Republic established and used methodic with ultrasound densitometry, which was helpful for primary risk group including decreasing of mineral density, disorders of bone architectonic and strength of skeletal bones.

Treatment methodic established by Obstetrics and Gynaecology Institute specialists included physical exercises and medical therapy. Physical exercises were prepared for all age groups. There was difference in intensity of exercises according to patient's age. Main task of exercises was strengthening of vertebral column.

Methods There was used non invasive ultrasound densitometer SUNLIGHT OMNISENSE 7000 included 3 probes for examination of different bones ( radius, tibia and phalanx of thumb) for diagnostic, screening and monitoring of osteopenia and osteoporosis. We have observed by densitometry 722 female patients (middle age 56, $4 \pm 1,0$ y.o.) trying to detect osteoporosis in postmenopausal syndrome stage on period from January 2009 till October 2014. There were $382(52,9 \%)$ cases with osteoporosis and $211(29,2 \%)$ cases with osteopenia. There weren't pathological process in $129(17,9 \%)$ cases.

Densitometry examination of patients was interpreted by special computer program. Computer program estimated patient's status as osteoporosis, osteopenia or normal; also computer program predicted percentage possibility of fracture. Re- examinations were arranged after 6 months and 1 year.

Results Patients were treated by Obstetrics and Gynaecology Institute approved method. There was used complex treatment method included medical therapy and physical exercises. Physical activity was indicated as important part of treatment. Physical exercises course continued 3 months. All patients didn't interrupt exercises after finish of treatment. Medical therapy includes hormonal therapy, $\mathrm{Ca}$ and bisphosphonates. Successful results of treatment demonstrated efficiency of treatment method and importance of physical activity in patients with osteoporosis and osteopenia.

Densitometry gave possibility to make monitoring during treatment period.

Conclusions Primary diagnostic of osteoporosis and osteopenia gave possibility to detect risk group and start complex treatment and dynamic monitoring. As result it was possible decrease clinical manifestation of osteoporosis and osteopenia to minimal level in limited period.

\section{POST TRAUMATIC STRESS DISORDERS AND QUALITY OF LIFE AMONG TRAFFIC CRASH SURVIVORS IN MALAYSIA}

'Kulanthayan S, 'S Sharifah, ${ }^{2}$ V Nathan. ${ }^{1}$ Universiti Putra Malaysia; ${ }^{2}$ Universiti Perdana

\subsection{6/injuryprev-2016-042156.789}

Background Traffic crashes (MVC) injure many people and affects victims' everyday lives and productivity. Traumatic experiences are common, but only a minority of survivors develops chronic emotional problems such as post-traumatic stress disorder (PTSD). The objective of this cross sectional study is to determine the quality of life (QoL) and PTSD among traffic crash survivors. Methods A total of 108 traffic crash survivors from the Orthopaedic Clinic of two leading public hospitals in Selangor, Malaysia (Serdang Hospital and Tengku Ampuan Rahimah Hospital) participated. Each respondent was interviewed face to face using WHOQOL-BREF questionnaire to measure QoL and Impact Event Scale (IES) to measure the level of PTSD.

Results The results found that $82.4 \%$ of the respondents developed PTSD and 56.5\% of the respondents had poor QoL. There is significant difference exists in social relationship domain with the level of PTSD ( $p<0.05$ ). There is also a significant difference between overall QoL score and the level of PTSD among the respondents $(p<0.05)$. The result also showed a significant difference between age $(p=0.044)$, race $(p=0.013)$ and educational level ( $p=0.002)$ with the mean score of QoL. However, there was no significant association between age, gender, marital status and educational level with the level of PTSD. QoL score was found to be significantly correlated with PTSD ( $p=0.030)$. Conclusions In conclusion, traffic crash involvement is a leading cause to PTSD. Decrements in QoL have been associated with both chronic pain and also PTSD. Hence, greater attentions need to be given for those traffic crash survivors who developed PTSD symptoms.

\section{A RANDOMISED CONTROL TRIAL STUDY ON BIOPSYCHOSOCIAL COACHING INTERVENTION}

Nurrul Hafeezah Sahak, Kulanthayan KC Mani. University Putra,Malaysia

\subsection{6/injuryprev-2016-042156.790}

Background Physical injury is one of the outcomes due to road traffic accidents. These survivors, who are left with temporary or permanent disability, may result in continuous restriction on their physical functioning which gives impact to their health related quality of life (HRQOL). The aim of this study was to evaluate the impact of a biopsychosocial intervention on HRQOL among road traffic accident survivors.

Methods A randomised control trial study design was applied among 200 respondents who were equally divided into the intervention and control group. All respondents in both groups were 
required to answer SF36 questionnaire at baseline to determine their HRQOL status after accident. However only those in the intervention group were given coaching, which was delivered concurrent to usual care. The effectiveness of this intervention was measured after all respondents in both groups had finished intervention and usual care respectively-they were required to answer SF36 questionnaire once again.

Results The follow up stage is still ongoing. However, the result will be ready at the time of this conference. Potential results are the mean of HRQOL will be reduced more significantly among those in the intervention group compare to those who are only receive usual care. Low self-esteem among these survivors will be improved as this intervention provides support to regain self-confidence that has lost due to the injury.

Conclusions We are absolutely convinced that the module in this intervention is able to assist injured person to improve their emotional wellbeing. Therefore the combination of biopsychosocial coaching intervention with usual care is believed to give synergistic effect to the positive changes on HRQOL status.

\section{INCIDENCE, COSTS AND OUTCOMES OF NON-UNION, DELAYED UNION AND MAL-UNION FOLLOWING LONG BONE FRACTURE}

${ }^{1}$ Christina L Ekegren, ${ }^{1}$ Belinda J Gabbe, ${ }^{1,2}$ Elton R Edwards, ${ }^{3,4}$ Richard de Steiger, ${ }^{5,6}$ Richard Page. ${ }^{1}$ Monash University, Australia; ${ }^{2}$ The Alfred Hospital, Australia; ${ }^{3}$ Epworth Healthcare, Australia; ${ }^{4}$ University of Melbourne, Australia; ${ }^{5}$ University Hospital Geelong, Australia; ${ }^{6}$ Deakin University, Australia

\subsection{6/injuryprev-2016-042156.791}

Background The management of long bone fractures is complex and the risk of healing complications persists. Previous studies have reported widely varying incidence and cost estimates for fracture healing complications, with the majority focusing on small cohorts with specific fracture types and treatment methods. Also, there is a lack of research on patient outcomes. The aim of this study was to describe the incidence, inpatient costs and 12month outcomes of readmissions for healing complications following long bone fracture.

Methods All humeral, tibial and femoral fractures registered by the Victorian Orthopaedic Trauma Outcomes Registry over a 5-year period were linked with hospital data to identify 2-year readmissions for non-union, delayed union or mal-union. Study outcomes for patients with complication readmissions included hospital length of stay, inpatient costs, function (Extended Glasgow Outcome Scale), work status and quality of life (EQ-5D) measured 12 months post-injury. Cases with and without complications were compared using univariable and multivariable methods.

Results Of the 3908 patients included, 9\% were readmitted for healing complications within 2 years of their index fracture. The most common complication type was non-union (77\% of complications). Admissions for fracture healing complications incurred an extra 3 days in hospital and costs of up to AUD $\$ 25,000$ per patient (AUD \$5.4 M in total). Patients with healing complications reported worse function, quality of life and return to work rates 12 months post-injury. After adjusting for key confounders, patients had higher odds of developing complications if they were older, receiving compensation or had multiple fractures.

Conclusions Patients who develop complications have poorer outcomes and place additional burden on healthcare and compensation systems. As such, there is a need to prioritise future research aimed at preventing fracture healing complications and improving patient outcomes.

\section{MANAGEMENT OF STRESS \& MUSCULOSKELETAL DISORDERS IN THE WORKPLACE BY YOGA, MEDITATION AND THERAPEUTIC REFLEXOLOGY}

'PK Goswami, ' 2 Samantha Jane Burnell. 'Labour Department, Government of Delhi; ${ }^{2} T$ The International Academy of Reflexology and Meridian Therapy, South Africa

\subsection{6/injuryprev-2016-042156.792}

Problem $\mathrm{n}$ the modern work environments of today, we have started putting strain on our systems as a whole- due to physical, mental and emotional demands far surpassing what our systems can bear. These exposures are going against the natural laws of nature and often done without conscious awareness of the longer-term damage. There is an extended list of occupational disorders that exist, nowadays, due to the upright skeletal position of the man, and more so because of the uninvited pressures that he has put onto the skeletal structure as a whole. The result equals the vertebral column being unable to withstand the stresses of prolonged standing or sitting down, especially when unsupported.

Description of problem Musculoskeletal disorders and diseases are common occupational problems all over the world. They are a result of the previous research of author "Health hazards in Small and Medium Enterprises garment industries in developing countries" has been taken for this further study. The previous study was conducted in Delhi from August-2014 to December2014. The database and result of the study with respect to Indian population has been chosen for this further study. The study revealed that musculoskeletal problems (54\%) were the commonest health problem in India. The objective of the study is to design an effective program to create an awareness surrounding the management of stress, Musculoskeletal problems as well as an effective management of pain related symptoms by using Yoga, meditation and Therapeutic Reflexology and Meridian Therapy.

Result After an intervention by Yoga and Meditation therapies for prevention and cure of Musculoskeletal problems, as well as Reflexology treatments for an on-going maintenance of immediate pain relief, it is found that $27 \%$ workers suffering from acute musculoskeletal problem and $83 \%$ workers suffering from moderate Musculoskeletal problems are no longer experiencing chronic symptoms or completely cured of pain. The Study period is January 2015 to July 2015.

Conclusion Yoga and Meditation is a philosophy and practice that connects the body, breath, and mind to energise and balance the whole system in our body and Reflexology works on the premise that disease is caused by blockages along the meridians, and therefore the treatment is conducted to increase the circulation within the body, clear congestions along the pathways and stimulate the body's own healing potential. Yoga and Meditation can be used for managing Musculoskeletal problems and furthermore, there is vast scope of research for inclusion of Reflexology and Meridian therapy sessions for immediate and maintained pain relief results in this program.

\section{ANALYSIS OF THE EMERGENCY MEDICAL SERVICES' RESPONSE TO ROAD TRAFFIC INJURIES IN MEXICO}

'Juan Daniel Vera-López, ${ }^{1}$ Ricardo Pérez-Núñez, ${ }^{2}$ Lourdes García-Gómez, ${ }^{2}$ Elisa HidalgoSolozano. 'Secretariado Técnico De Consejo Nacional Para La Prevención De Accidentes, México D.F; ${ }^{2}$ National Institute of Public Health of Mexico

10.1136/injuryprev-2016-042156.793 